\title{
Vulvar Cancer by AJCC v6 Stage
}

National Cancer Institute

\section{Source}

National Cancer Institute. Vulvar Cancer by A/CC v6 Stage. NCI Thesaurus. Code C90345.

A term that refers to the staging of vulvar carcinoma according to the American Joint Committee on Cancer, 6th edition. 